International Journal of Advancement in Life Sciences Research

Online ISSN: 2581-4877

journal homepage http://ijalsr.org

Review Article

\title{
Influence of Oral Microbiome on Human Health: an Overview
}

\author{
Mitu De ${ }^{1, \star}$, Manisha Bhattacharya ${ }^{2}$, Animita C. Saha ${ }^{3}$, Indrani Basu ${ }^{4}$, Sayak Ganguli ${ }^{5}$ and \\ Santi Ranjan Dey ${ }^{6}$ \\ ${ }^{1}$ Associate Professor, Department of Botany, Gurudas College, Kolkata 54 \& Head of Research and Academic \\ Studies Unit (Hony), Autism Society West Bengal, Kolkata, India \\ ${ }^{2}$ Clinical Psychologist, Autism Society West Bengal, (ASWB), Kolkata, India \\ ${ }^{3}$ Associate Professor of Medicine, University of North Carolina School of Medicine, Chapel Hill, NC, USA \\ ${ }^{4}$ Director, Autism Society West Bengal, Kolkata, India \\ ${ }^{5}$ Group Leader, Amplicon Institute of Interdisciplinary Science and Technology, Palta, West Bengal, India \\ ${ }^{6}$ Assistant Professor, Department of Zoology, Rammohan College, Kolkata, India. \\ ${ }^{*}$ Correspondence E-mail : mitude@rediffmail.com
}

\begin{abstract}
The human microbiome can be considered a counterpart of the human genome. The genes in our microbiome outnumber the genes in the human genome by about 100 to 1 Advances in DNA sequencing technologies have created a new field of research, called metagenomics. . Microbiome projects worldwide have been launched with the goal of understanding the roles that these symbionts play and their impacts on human health. The advent of next-generation sequencing (NGS) technologies and their applications on human populations have led to the identification of a large catalog of rare and common variants. The Human Microbiome Project (HMP) has analysed the largest cohort and set of distinct, clinically relevant body habitats so far. The richest area in terms of total numbers of microbes in the body is the human gut. The relationship between the oral microbiome and its host is dynamic. The oral microbiome is also considered a key cause of oral diseases, including dental caries and periodontal diseases, as well as many systemic diseases such as diabetes and cardiovascular diseases and disorders like Autism Spectrum Disorders (ASD). Metagenomics and metabolomics based reserach is providing a multidimensional picture of the microbiome community, revealing its genetic potential and metabolic activities. In this paper an overview is given on metagenomics and the human oral microbiome.
\end{abstract}

Keywords: Human Microbiome Project, Oral microbiome, Metagenomics

\section{Introduction}

Human beings live in symbiosis with a vast population of bacteria, archaea, eukaryotes, and viruses, collectively called microbiota, that colonize different bodily parts. Human beings can be considered as "superorganisms" as a result of their close symbiotic associations with the gut microbiota (Lederberg, 2000). The human microbiota consists of the 10-100 trillion symbiotic microbial cells harbored by each person, primarily bacteria in the gut. The human microbiome consists of the genes these cells harbour (Turnbaugh et al., 2007). The human microbiome can be considered a counterpart of the human genome. The genes in our microbiome outnumber the genes in the human genome by about 100 to 1 . The human 
microbiome, or community of microbes and collection of genomes found in and on the human body, is now the subject of renewed, intense study (Relman, 2012).

Because of their small size, however, microorganisms make up only about 1 to 3 percent of our body mass. Superorganism metabolism involves integration of truly indigenous metabolic processs (coded in the host genome) with those of the microbiome. These results in extensive transgenomic cometabolism of many substrates including those involved in host metabolic regulation (Nicholson et al., 2005). The superorganism concept represents an important paradigm shift in understanding human biology and is likely to have a significant impact on the future of disease prevention and therapy (Nicholson, 2006). Recent works have shown that the exact human microbiome composition varies between healthy people (Turnbaugh et al, 2009)

\section{Human Microbiome and Human Health}

Although the human microbiome has long been known to influence human health and disease (Qin et al, 2010; Relman, 2012), only recently researchers have begun to appreciate the breadth of its involvement.

The Human Microbiome Project has analysed the largest cohort and set of distinct, clinically relevant body habitats so far. It was seen that the diversity and abundance of each habitat's signature microbes tend to vary widely even among healthy subjects, with strong niche specialization both within and among individuals. The project encountered an estimated $81-99 \%$ of the genera, enzyme families and community configurations occupied by the healthy Western microbiome (HMP et al., 2012).

This is almost entirely due to the recent ability of high-throughput sequencing to provide an efficient and cost-effective tool for investigating the members of a microbial community and how they change. Dysfunctions of the human microbiota are increasingly being linked to several diseases and disorders. Modern highthroughput sequencing and bioinformatic tools provide a powerful means of understanding the contribution of the human microbiome to health and its potential as a target for therapeutic interventions.

\section{Metagenomics}

Advances in DNA sequencing technologies have created a new field of research, called metagenomics, allowing comprehensive examination of microbial communities, without the need for cultivation. Instead of examining the genome of an individual bacterial strain that has been grown in a laboratory, the metagenomic approach examines the collection of genomes derived from microbial communities sampled from natural environments.

These revolutionary advances in genomics and associated technologies, and in computational biology, are another factor that explains current attention to the human microbiome (Relman, 2011). Greatly improved capabilities in DNA sequencing have enabled microbial community molecular phylogenetic surveys, and metagenomic and metatranscriptomic analyses of clinical specimens, to far greater depth, at far greater speed, and at far less cost. Metagenomics and metabolomics provide a more rich, textured, and multidimensional picture of the community, revealing its genetic potential and metabolic activities (Turnbaugh and Gordon, 2008; Turnbaugh et al, 2009; Holmes et al, 2011). Tools developed in other fields of science for pattern recognition and identification of predictive features in complex, multi-dimensional data are now being applied to genomic data from the human microbiome (Reshef et al., 2011).

The advent of culture-independent methods has greatly improved the detection of microorganisms, many of which cannot yet be grown in culture (Zarco, 2012). The most common culture-independent technique to analyse the microbiome is based on $16 \mathrm{~S}$ ribosomal RNA (16S rRNA) gene community profiling (Zarco, 2012). The 16S rRNA gene is present in all prokaryotes and contains variable regions that are unique between microorganisms and that can be used as a means of identification. 16S rRNA genes can be extracted from heterogeneous samples, amplified and sequenced, and then compared with databases such as the Human 
Oral Microbiome Database, a publically available online resource that links sequence data with phenotypic, phylogenetic, clinical and bibliographic information of microorganisms found in the oral cavity (HOMD, 2016).

The advent of nextgeneration sequencing (NGS) technologies and their applications on human populations have led to the identification of a large catalog of rare and common variants (Tennessen, et al.2012; Auton, et al. 2015). The use of NGS on large isolated population cohorts (Gudbjartsson 2015 et al.) and on specific disease cohorts has led to the discovery of a number of new disease/disorder genes like Autism Spectrum Disorder (ASD).

\section{Microbiomics}

'Microbiomics' is a fast-growing field in which all the microorganisms of a given community (a 'microbiota') are investigated together. This could be the microbiota of an environmental sample (e.g. soil or water), a particular body site (e.g. the gut or the mouth) or from a particular organism (e.g. farm or zoo animals). Investigation of environmental microbial communities can be of particular interest in discovery of 'novel' organisms with exciting properties, such as production of natural products with antimicrobial properties.

Microbiomics can consort personalized medicine to revolutionize patient treatment modalities (Shukla et al, 2015), in an era when non-communicable disease is a burden compared to waning communicable disease (Ebrahim and Smeeth, 2006; Barouki et al, 2012; Prescott, 2014). The advent of next generation sequencing (NGS) or highthroughput sequencing has revolutionized the field of microbiome analysis, providing the tools necessary to address the issue (Gevers et al, 2012). This prompted the launch of the NIH's Human Microbiome Project (HMP), constructed as a large, genome-scale community research project.

\section{Human Microbiome Project (HMP)}

Microbiome projects worldwide have been launched with the goal of understanding the roles that these symbionts play and their impacts on human health (Peterson et al, 2009; Qin et al., 2010)

Worldwide research initiatives are mapping the human microbiome, giving insight into uncharted species and genes. One initiative is the Human Microbiome Project (HMP) sponsored by the National Human Genome Research Institute (NHGRI), part of the National Institutes of Health (NIH). The purpose of the HMP is to study the human as a supraorganism composed of non-human and human cells, with the goal of describing the human microbiome and analyzing its role in human health and disease.

A major goal of the HMP is to characterize the metagenome (the combined genomes of all the microbes) of the microbiomes of 300 healthy people, over time. Different body sites support entirely distinct microbial communities due to differences in prevailing biological and physical properties of each location/habitat (Costello et al., 2009).

Five major body areas are sampled: Skin, mouth, nose, colon, and vagina.Women were sampled at 18 body habitats, men at 15 (excluding three vaginal sites), distributed among five major body areas. Nine specimens were collected from the oral cavity and oropharynx: saliva; buccal mucosa (cheek), keratinized gingiva (gums), palate, tonsils, throat and tongue soft tissues, and supra- and subgingival dental plaque (tooth biofilm above and below the gum). Four skin specimens were collected from the two retroauricular creases (behind each ear) and the two antecubital fossae (inner elbows), and one specimen for the anterior nares (nostrils). A self-collected stool specimen represented the microbiota of the lower gastrointestinal tract. Based on the data The Human Microbiome Project Consortium published a research paper in the journal Nature in 2012 on the Structure, function and diversity of the healthy human microbiome. (HMP et al., 2012).

The richest area in terms of total numbers of microbes in the body is the human gut. A review article in the journal Science in a special section appropriately titled "The Inner Tube of Life" had this to say:

"The adult human gastrointestinal (GI) tract contains all three domains of life-bacteria, 
archaea, and eukarya. Bacteria living in the human gut achieve the highest cell densities recorded for any ecosystem."

Our gut microbiota can be pictured as a microbial organ placed within a host organ: It is composed of different cell lineages with a capacity to communicate with one another and the host; it consumes, stores, and redistributes energy; it mediates physiologically important chemical transformations; and it can maintain and repair itself through self-replication. The gut microbiome, which may contain > 100 times the number of genes in our genome, endows us with functional features that we have not had to evolve ourselves (Bäckhed et al., 2005).

\section{Oral Microbiome}

The term "gut or oral microbiome" refers to the community of microorganisms that inhabit the gut, and includes both helpful and harmful bacteria. So Oral microbiome, by definition, is the collective genomes of microorganisms that reside in the oral cavity (Kolenbrander et al, 2002; Turnbaugh et al, 2007). Many researchers believe that the characterisation of oral microbiome is an essential step in understanding oral health and systemic diseases (Dewhirst et al., 2010).

One of the most clinically relevant microbial habitats, the human oral cavity is colonized by a personalized set of microorganisms, including bacteria, archaea, fungi, and viruses (Wade, 2013). The oral microbiome is also considered a key cause of oral diseases, including dental caries and periodontal diseases, as well as many systemic diseases such as diabetes and cardiovascular diseases (He et al, 2015; Zarco et al., 2012) and disorders like Autism Spectrum Disorders (ASD) (Hicks et al., 2018). Because of its crucial role in oral and systemic health, the oral microbiome has become an essential part of microbiomics.

The acquisition of microbiota continues over the first few years of life, as an infant's Gl tract microbiome begins to resemble that of an adult as early as 1 year of life (Palmer, 2007). The gastrointestinal (GI) tract of a human infant provides a brand new environment for microbial colonization (Mackie et al., 1999).
The use of fire, invention of agriculture, increased access to processed foods, including refined sugar after the industrial revolution, and the advent of antimicrobial therapy, are all likely to have influenced the composition of the human microbiome (Gillings et al., 2015).

\section{Concept of the microbiota-gut-brain axis}

The concept of the gut-brain axis, a bidirectional channel of communication between the "big brain" in the cranium and the "little brain" (i.e. the enteric nervous system) was introduced to describe the central and peripheral effects of gut-brain peptides such as cholecystokinin and bombesin (Banks). Recently, connections between the gut and the brain have been extended to include a new participant; the microbiota leading to the concept of the microbiota-gut-brain axis. The gut harbours an enormous diversity of microbes that are essential for the maintainance of homeostasis in health and disease (Marcel et al., 2017). The role of the gut or oral microbiota in the regulation of physiology in both health and disease is becoming increasingly recognized (Marchesi et al, 2016; Goulet, 2015). Several papers analyzed the HMP oral datasets (Segata et al., 2012; Huse et al., 2012; Li et al., 2013) revealing great variability of the oral microbiome among and within healthy individuals.

\section{Relationship between the oral microbiome and its host}

The microorganisms found in the human oral cavity have been referred to as the oral microflora, oral microbiota, or oral microbiome (Dewhirst et al., 2010). The relationship between the oral microbiome and its host is dynamic and, while in the healthy mouth the composition of microbial communities is remarkably stable (after the microbiome has matured in childhood), biological changes in a person's life can affect the balance of the species within these communities (Marsh et al, 2015). Evidence has emerged from animal models to suggest that bacteria resident in the gut could impact on the "big brain" and even contribute to neurological and neuropsychiatric disease. 
Recent research has shown that the microorganisms that make up the human microbiome are not just unicellular organisms living alongside each other, but instead form highly regulated, structurally and functionally organised communities attached to surfaces as biofilms with interspecies collaborations as well as antagonisms that contribute to ecologic stability ( $\mathrm{Li}$ and Tian, 2012). It has been reported that more than 700 prokaryotic taxa have been detected in the oral cavity, many of which cannot be isolated by common culture methods (HOMD, 2016). Approximately 54\% are validly named species, $14 \%$ are unnamed (but cultivated) and $32 \%$ are known only as uncultivated phylotypes.

The microbiome has emerged as a potential therapeutic target in disorders as diverse as IBS, Parkinson's disease and depression (Felice et al., 2016; Yarandi et al., 2016). Many other neuropsychiatric disorders including Alzheimer's disease, amyotrophic lateral sclerosis, autism, stroke and drug

\section{References}

Auton, A. et al. (2015). A global reference for human genetic variation. Nature, 526 , pp. 68-74

Bäckhed F, et al. (2005). Host-Bacterial Mutualism in the Human Intestine. Science. 307 (5717), pp. 1915-1920.

Barouki Robert., et al. (2012). "Developmental Origins of Non-Communicable Disease: Implications for Research and Public Health". Environmental Health. 11, pp. 42.

Costello E K, Lauber C L, Hamady M et al. (2009). Bacterial community variation in human body habitats across space and time. Science; 326 : 1694-1697.

Dewhirst FE, Chen T, Izard J, Paster BJ, Tanner AC, Yu WH, Lakshmanan A, Wade WG. (2010). The human oral microbiome. J Bacteriol. 192 (19), pp.5002-5017.

Ebrahim Shah \& Liam Smeeth. (2006). "NonCommunicable Diseases in Low-Income and Middle-Income Countries: a Debate?" International Journal of Epidemiology. 35(2), pp. 494-495.

Felice, V.D., Quigley, E.M., Sullivan, A.M., O’Keeffe, G.W., O'Mahony, S.M. (2016). Microbiota-Gut-Brain signalling in Parkinson's disease: Implications for Non-Motor Symptoms. Parkinsonism Relat. Disord. 27, pp.1-8.

Gevers, D., Pop, M., Schloss, P.D., Huttenhower, C. (2012). Bioinformatics for the Human Microbiome Project. PLoS Comput Biol. 8, e1002779. addiction have been linked, in one way or another, to the microbiome (Quigley, 2017).

\section{Conclusion}

Throughout human evolution, our environment has continuously shaped the composition of our microbiome, increasingly so during Neolithic, industrial revolution and modern eras. The sequencing of the human genome has driven the study of human biology in a significant way and enabled the genome-wide study to elucidate the molecular basis of complex human diseases and disorders. The role of microbiome on human physiology and health has received much attention in recent research. A number of human disorders and diseases have been directly and indirectly associated with the microbiome. Metagenomics and metabolomics based reserach is providing a multidimensional picture of the microbiome community, revealing its genetic potential and metabolic activities.

Gillings, M. R., Paulsen, I. T., Tetu, S. G. (2015). Ecology and evolution of the human microbiota: Fire, farming and antibiotics. Genes (Basel). 6, pp. 841-857.

Goulet, O. (2015). Potential role of the intestinal microbiota in programming health and disease. Nutr Rev. 73 (Suppl. 1), pp.32-40.

He, J., Li, Y., Cao, Y., Xue, J., Zhou, X. (2015). The oral microbiome diversity and its relation to human diseases. Folia Microbiol (Praha). 60, pp.69-80.

Hicks, S.D., Uhlig, R., Afshari, P., Williams, J., Chroneos, M., Tierney-Aves, C., Wagner, K., Middleton, F.A. (2018). Oral microbiome activity in children with autism spectrum disorder. Autism Research. 11 (9), pp. 1286-1299.

Holmes, E., Li, J.V., Athanasiou, T., Ashrafian, H., Nicholson, J.K. (2011). Understanding the role of gut microbiome-host metabolic signal disruption in health and disease. Trends Microbiol. 19(7), pp. 349-359.

The Human Microbiome Project Consortium. (2012). Structure, function and diversity of the healthy human microbiome. Nature. 486, pp.207-214.

Huse, S.M., Ye, Y., Zhou, Y., Fodor, A.A. (2012). A core human microbiome as viewed through $16 \mathrm{~S}$ rRNA sequences clusters. PLoS One. 7:e34242.

Kolenbrander, P.E., Andersen, R.N., Blehert, D.S. et al. (2002). Communication among Oral Bacteria. Microbiol Mol Biol Rev. 66, pp.486-505. 
Lederberg J. (2000). Infectious history.Science. 288, pp.287-293.

Li, K., Bihan, M., Methé, B.A. (2013). Analyses of the stability and core taxonomic memberships of the human microbiome. PLoS One. 8, e63139.

Li, Y. H. \& Tian, X. (2012). Quorum sensing and bacterial social interactions in biofilms. Sensors (Basel). 12, pp. 2519-2538.

Mackie, R.I., Sghir, A., Gaskins, H.R. (1999). Developmental microbial ecology of the neonatal gastrointestinal tract. Am J Clin Nutr. 69, pp.1035S-1045S.

Marsh, P.D., Head, D. A., Devine, D. A. (2015). Ecological approaches to oral biofilms: control without killing. Caries Res. 49 (Suppl 1), pp. 46-54.

Marcel van de Wouw, Schellekens, H., Timothy, G. Dinan, Cryan, J. F. (2017). Microbiota-Gut-Brain Axis: Modulator of Host Metabolism and Appetite, The Journal of Nutrition. 147,(5), pp. 727745.

Marchesi, J.R., Adams, D.H., Fava, F., Hermes, G.D., Hirschfield, G.M., Hold, G., Quraishi, M.N., Kinross, J., Smidt, H., Tuohy, K.M. et al. (2016). The gut microbiota and host health: a new clinical frontier.Gut. 65, pp. 330-9.

Nicholson, J.K. (2006). Global systems biology, personalized medicine and molecular epidemiology. Mol Syst Biol. 2, pp.52.

Nicholson, J.K., Holmes, E., Wilson, I.D. (2005). Gut microorganisms, mammalian metabolism and personalized health care. Nat Rev Microbiol. 3, pp.431-438.

Palmer, C., Bik, E.M., DiGiulio, D.B., Relman, D.A., Brown, P.O. (2007). Development of the human infant intestinal microbiota. PLoS Biol. 5, pp. e177.

Peterson, J., Garges, S., Giovanni, M., Mclnnes, P., Wang, L., Schloss, J.A., Bonazzi, V., McEwen, J.E., Wetterstrand, K.A., Deal, C., et al. (2009). The NIH Human Microbiome Project. Genome Research. 19, pp.2317-2323.

Prescott, Susan, L. (2014). "Disease Prevention in the Age of Convergence - the Need for a Wider, Long Ranging and Collaborative Vision". Allergology International, 63(1), pp.11-20.

Qin, J., Li, R., Raes, J., Arumugam, M., Burgdorf, K.S., et al. (2010). A human gut microbial gene catalogue established by metagenomic sequencing. Nature. 464, pp.59-65.
Int J Adv Life Sci Res. Volume 2(1)16-21 Quigley, E.M.M. (2017). Microbiota-Brain-Gut Axis and Neurodegenerative Diseases. Curr. Neurol. Neurosci. Rep. 17, pp.94.

Relman, David A. (2012). The human microbiome: ecosystem resilience and health. Nutr Rev. 70(Suppl 1), pp.S2-S9.

Relman, D.A. (2011). Microbial genomics and infectious diseases. N Engl J Med. 365(4), pp.347357.

Reshef, D.N., Reshef, Y.A., Finucane, H.K., et al. (2011). Detecting novel associations in large data sets. Science. 334(6062), pp.1518-1524.

Segata, N., Haake, S.K., Mannon, P., et al. (2012). Composition of the adult digestive tract bacterial microbiome based on seven mouth surfaces, tonsils, throat and stool samples. Genome Biol. 13, R42. doi: 10.1186/gb-2012-13-6-r42.

Shukla, S. K., et al. (2015). "Personalized Medicine Going Precise: from Genomics to Microbiomics". Trends in Molecular Medicine. 21(8), pp.461-462.

Tennessen, J.A. et al. (2012). Evolution and functional impact of rare coding variation from deep sequencing of human exomes. Science. 337, pp. 64-69.

Turnbaugh, P.J., Hamady, M., Yatsunenko, T., et al. (2009). A core gut microbiome in obese and lean twins. Nature. 457(7228), pp. 480-484.

Turnbaugh, P.J., Gordon, J.I. (2008). An invitation to the marriage of metagenomics and metabolomics. Cell. 134(5), pp.708-713.

Turnbaugh, P.J., Ley, R.E., Hamady, M., FraserLiggett, C.M., Knight, R., Gordon, J.I. (2007). The human microbiome project. Nature. 449, pp.804810.

Wade, W.G. (2013). The oral microbiome in health and disease. Pharmacol Res. 69, pp.137-143.

Yarandi, S.S., Peterson, D.A., Treisman, G.J., Moran, T.H., Pasricha, P.J. (2016). Modulatory effects of gut microbiota on the central nervous system: How the gut could play a role in neuropsychiatric health and disease. J. Neurogastroenterol. Motil. 22, pp. 201-212.

Zarco, M. F., Vess, T. J., Ginsburg, G. S. (2012). The oral microbiome in health and disease and the potential impact on personalized dental medicine. Oral Dis. 18, pp.109-120. 